Revista de Psicología Vol. 36 (1), 2018 (ISSN 0254-9247)

\title{
Propiedades psicométricas de la Escala de Creencias Acerca del Proceso Educativo en el Medio Rural (CAPE-R) ${ }^{1}$ en docentes de Mendoza, Argentina
}

\author{
Agustina Labin ${ }^{2}$, Paula Canizzo ${ }^{3}$, Leandro Casari ${ }^{4}$, Valeria Estefanía Moran ${ }^{5}$ \\ Universidad Nacional de San Luis², Dirección General de Escuelas, Mendoza \\ Pontificia Universidad Católica Argentina ${ }^{4}$, Centro de Investigaciones de \\ la Facultad de Psicología (CIPSI), Universidad Nacional de Córdobas, \\ CONICET $T^{2,4,5}$, Argentina
}

El artículo tuvo los siguientes objetivos, analizar la estructura psicométrica de la escala CAPE-R que evalúa las creencias acerca del proceso educativo en docentes rurales, conocer el perfil docente y analizar si existen diferencias significativas entre las creencias según variables sociodemográficas. Se trabajó con una muestra de 150 docentes rurales de Mendoza, Argentina. Los resultados del análisis factorial exploratorio arrojaron una estructura factorial conformada por cuatro factores que explicaron un $44.19 \%$ de la varianza de respuestas al test. Con respecto a la consistencia interna, los coeficientes oscilaron entre .74 y .85 . Los datos muestran un predominio de la dimensión dificultades en el proceso educativo $(M=2.83 ; D E=.69)$. Además, es la única dimensión que alcanzó una asociación significativa con el nivel educativo en el que el docente imparte clases $(F=4.77, p<.05)$.

1 Los autores agradecen a Daniela Vera Bachmann y Paulina Meneses Clavero, creadoras de la Escala CAPE-R

2 Licenciada en Psicología. Becaria doctoral Consejo Nacional de Investigaciones Científicas y Técnicas (CONICET). Docente de la Facultad de Psicología de la Universidad Nacional de San Luis. Dirección postal: Ejército de Los Andes 950, D5700HHW, Box 26, Facultad de Psicología, UNSL. Contacto: alabin@unsl.edu.ar

3 Licenciada en Psicología. Psicóloga en Salud Laboral de la Dirección General de Escuelas de Mendoza, Argentina. Dirección postal: Peltier 351, 5500, Mendoza. Contacto: pau.canizzo@ gmail.com

4 Doctor en Psicología. Becario postdoctoral Consejo Nacional de Investigaciones Científicas y Técnicas (CONICET). Docente de la Universidad Católica Argentina. Dirección postal: Av. Dr. Ruiz Leal s/n. Parque General San Martín. INCIHUSA - CONICET CCT Mendoza. Mendoza (5700), Argentina. Contacto: leandro_casari@uca.edu.ar

5 Doctora en Psicología. Becaria postdoctoral Consejo Nacional de Investigaciones Científicas y Técnicas (CONICET). Profesora en la Universidad Católica de Cuyo y Universidad de Mendoza. Dirección postal: LACI, Facultad Psicología, Universidad Nacional de Córdoba, Ciudad Universitaria, Córdoba. Contacto: moranvaleria@gmail.com 
Palabras clave: creencias, docentes rurales, proceso educativo, perfil docente, CAPE-R.

Psychometric properties of the Belief about the Educational Process in the Rural Environment Scale (CAPE-R) in teachers from Mendoza, Argentina

This study analyzes the psychometric structure of the CAPE- R scale, which assesses the beliefs about the educational process in rural teachers; explores the teacher's profile, and analyzes the significant differences between the beliefs according to sociodemographic variables. The sample included 150 rural teachers from Mendoza, Argentina. The exploratory factor analysis yielded a factorial structure made up of four factors that explained a $44.19 \%$ of the variance of responses to the test. Internal consistency coefficients ranged from .74 to .85 . The data show a predominance of difficulties in the educational process dimension $(M=2.83 ; D E=.69)$, the only dimension that reached a significant association with the educational level of the classes $(F=4.77, p<.05)$.

Keywords: beliefs, rural teachers, educational process, teacher profile, CAPE-R.

\section{Propriedades psicométricas da Escala de Crenças acerca do processo educacional nas áreas rurais (CAPE-R) professores em Mendoza, Argentina}

$\mathrm{O}$ artigo teve os seguintes objetivos, analisar a estrutura psicométrica da escala CAPE-R que avalia as crenças sobre o processo educativo em professores rurais, conhecer o perfil docente y analisar se existem diferenças significativas entre as crenças a partir de variáveis sociodemograficas. Trabalhou-se com uma amostra de 150 professores rurais de Mendoza, Argentina. Os resultados da análise fatorial exploratória rendeu uma estrutura fatorial composto por quatro fatores a $44.19 \%$ explicou que da variaçáo de respostas para o teste. No que diz respeito à consistência interna, os coeficientes variou ,74-.85. Os dados mostram uma predominância de dificuldades na dimensão processo de ensino $(H=2.83 ; D P$ : .69). Além disso, é a única dimensão que atingiram uma associação significativa com o nível educacional em que o professor ensina $(F=4.77, p<.05)$.

Palavras chave: crenças, professores rurais, processo educativo, perfil docente, CAPE-R

Propriétés psychométriques de l'échelle des croyances sur le processus éducatif en milieu rural (CAPE-R) chez les enseignants de Mendoza, Argentine

Les objectifs de l'article étaient d'analyser la structure psychométrique de l'échelle des croyances sur le processus éducatif en milieu rural, de connaître le profil d'enseignement et d'analyser s'il existe des différences significatives entre les croyances sociodémographiques. Nous avons travaillé avec un échantillon de 150 enseignants ruraux de Mendoza, en Argentine. Les résultats de l'analyse factorielle exploratoire ont donné une structure factorielle composée de quatre facteurs expliquant $44,19 \%$ de la variance des réponses au test. En ce qui concerne la cohérence interne, les coefficients variaient entre .74 et .85 . Les données montrent une prédominance de la dimension des difficultés dans le processus éducatif $(M=$ $2.83, S D=.69)$. En outre, c'est la seule dimension qui a atteint une association significative avec le niveau d'éducation dans lequel l'enseignant enseigne $(F=4.77, p<.05)$.

Mots-clés: croyances, enseignants ruraux, processus éducatif, profil de l'enseignant, l'échelle des croyances sur le processus éducatif en milieu rural. 
En la Argentina, se entiende a la docencia rural como prometedora y facilitadora de la transmisión de los contenidos culturales y es considerada como un servicio a la comunidad. Los docentes frecuentemente afrontan en soledad la toma de decisiones que su tarea exige y no suelen tener oportunidades de participar en instancias de encuentro con otros colegas (Blanco, 2007). Actualmente la tarea docente, en sentido amplio, implica una serie de demandas habituales que exceden cualquier noción clásica acerca del enseñar. De este modo, está sujeta a ser interpretada como un activismo aislado en tanto no posea un reconocimiento social e institucional que valore los saberes y procedimientos que se plasman a diario en las instituciones educativas. A su vez, se debe considerar el malestar, la extenuación, la flexibilización que implican los cambios en el rol docente que no cuenta con un encuadre delimitado de accionar (Duschatzky, 2007; Molpeceres, Chulvi \& Bernad, 2004; Rosbaco, 2007; Schiavetta, 2013).

En este contexto, los docentes rurales trabajan en comunidades aisladas, reciben matrículas reducidas, insuficientes para conformar un grupo por año de escolaridad. Asimismo, para entrar en la categoría de escuelas rurales se debe cumplir con un criterio pedagógico-legal alternativo de organización institucional con el fin de asegurar la trayectoria de sus alumnos, contar con agrupaciones de estudiantes en plurigrados, pluriańos, secciones múltiples, entre otras. Específicamente, en Mendoza (Argentina), las escuelas rurales son aquellas que están localizadas a más de 1 kilómetro del borde del mosaico principal de la localidad y cuya área de influencia es de 1000 metros compuestos por parcelas rurales, zonas naturales (montaña, bañado, cauce de ríos) en espacios geográficos con escasa disponibilidad de servicios públicos y medios de acceso limitados, entre otros aspectos (Dirección General de Cultura y Educación, 2007). En este marco, la docencia rural merece un apartado diferente ya que posee ciertas características propias con relación a su rol. Vera 
Bachmann y Meneses Clavero (2012) conciben al docente rural como un facilitador del proceso de aprendizaje, que conlleva una labor abierta, creativa, en la medida de lo posible que responda a los requerimientos de su medio. En otras palabras, un creador de espacios favorables para una auténtica participación comunitaria. Además, afirman que el rol del docente rural no se reduce, puntualmente, a cumplir las pautas de un programa educativo de aplicación general por lo cual, su actividad no solo se circunscribe a la relación alumno-profesor (Blanco, 2007; Castellanos Simons et al., 2015; Vera Bachmann, Osses \& Fuenzalida, 2012).

La actividad docente lleva a repensar en la diversidad de roles y peculiaridades que implica la ruralidad en sí misma, así como también en las características personales y las creencias educativas que impactan en la tarea docente y, en consecuencia, en el proceso educativo. Los docentes construyen sus creencias como resultado de un múltiple abanico de experiencias, que incluyen aspectos conscientes-inconscientes, personales-escolares, relacionales-individuales, los cuales conllevan a la conformación de expectativas y prejuicios difíciles de remover (Molpeceres et al., 2004). Las creencias se forman tempranamente, aluden a comprensiones, supuestos, imágenes, entre otras y tienden a autoperpetuarse. Persisten frente a la contradicción causada por el tiempo, la razón, la escolaridad o la experiencia. Generalmente, permanecen inalterables y acompañan a los docentes permanentemente durante sus labores de enseńanza, sin ser reflexivos acerca de su sistema de creencias y sin cuestionarse sobre cómo es que estas influyen en su práctica (Mansilla Sepúlveda \& Beltrán Véliz, 2013; Netzahualcoyotl-Netzahual, 2015; Prieto, 2008).

De este modo, evidenciar las creencias docentes propicia una oportunidad de reflexión que posibilitará indagar desde dónde llevan a cabo los docentes rurales sus prácticas educativas y cuáles son las influencias que estas pueden tener en los resultados de sus alumnos. El cambio de concepciones sobre el proceso de enseńanza-aprendizaje requiere un arduo ejercicio y es esencial para lograr un proceso de autoevaluación y análisis que perdure a través del tiempo (Contreras Palma, 2008; Molpeceres et al., 2004; Vera Bachmann et al., 2012). 
Si bien, aun no se ha llegado a establecer una única definición de las creencias, varios autores coinciden en el origen sociocultural que tienen las mismas y que, a su vez, son un factor de socialización (López Vargas \& Basto Torrado, 2010; Rodrigo, Rodríguez \& Marrero, 1993). En esta línea, se afirma que son una herencia cultural, resultado de cómo se han organizado las actividades de aprendizaje y enseñanza a lo largo de los años, la historia cultural del aprendizaje como actividad social y sobre todo de las propias prácticas docentes cotidianas (Pozo Municio et al., 2006). En este sentido, los docentes están guiados por sus creencias a la hora de evaluar, clasificar, e inclusive en su accionar pedagógico (Díaz, Martínez, Roa \& Sanhueza, 2010; Fernández Nistal, Pérez Ibarra, Tuset Bertrán \& García Hernández, 2013; Mansilla Sepúlveda \& Beltrán Véliz, 2013; Prieto, 2008). En coincidencia, Myers (2000), sostiene que las caracterizaciones que los docentes formulan sobre los estudiantes se correlacionan con los logros de estos. A su vez, en la práctica, estas creencias pueden tomar múltiples formas; puede que estén incluidas en las expectativas del profesor sobre el desempeño de algún alumno o en las teorías implícitas del docente acerca de un área o tema particular que está enseñando (Vera Bachmann et al., 2012). Más aún, los procesos de pensamiento serían los precursores y creadores de las creencias, sin embargo, el efecto filtro de las creencias, permite tanto interpretar la realidad, como redefinir, distorsionar y reestructurar el procesamiento posterior de la información (Díaz et al., 2010).

En la Argentina, Fairstein y Pedernera (2007) investigaron en dos escuelas rurales las concepciones docentes sobre el aprendizaje y la enseñanza mediante entrevistas semiestructuradas individuales a los docentes, registros de clase que consistían en observaciones no participantes, consulta de documentación escolar como el proyecto educativo institucional y las planificaciones didácticas tanto anuales como periódicas. En este estudio seńalaron la importancia de la docencia rural de múltiples grados, como una modalidad pedagógica particularmente diferente a las escuelas urbanas. Según los autores, en la escuela rural no existe un tiempo limitado convencional —-marcado habitualmente por el timbre-, existe autonomía y flexibilidad docente para 
responder a las diversas demandas de sus estudiantes, así como atención más personalizada hacia cada uno de ellos.

El reconocimiento de las concepciones sobre el aprendizaje requiere de un arduo ejercicio, develar las creencias es esencial para que se logre un proceso de autoevaluación y revisión que perdure a través del tiempo. Tanto conocer como estudiar las creencias propias de los docentes rurales posibilitará la trasformación del quehacer docente en el aula, como así también generar una nueva perspectiva para repensar la práctica pedagógica, su sentido y sus efectos, sobre todo de aquellos que provienen de los sectores más vulnerables (Contreras Palma, 2008; Guadagni \& Boero, 2015; Molpeceres et al., 2004; Vera Bachmann et al., 2012).

Vera Bachmann y Meneses Clavero (2012) encontraron imprescindible indagar las creencias que sustentan los docentes rurales y reconocer la influencia que tenían dichas creencias en las percepciones y juicios que realizan en el desempeño académico de los alumnos, sobre todo de aquellos que provienen de los sectores rurales. Así, diseñaron la Escala de Creencias Acerca del Proceso Educativo en el Medio Rural (CAPE-R) con el objetivo de evaluar el grado de acuerdo que el examinado manifiesta en relación a una serie de afirmaciones que representan creencias con respecto a distintos aspectos del proceso educativo en el medio rural. La creación de la CAPE-R, se enmarca dentro de las escasas investigaciones publicadas sobre las creencias de los docentes rurales y, proponen así una proximidad al rol del docente rural. La investigación, evidenció que la dimensión de creencias aprendizaje significativo obtuvo puntajes superiores. Esta conclusión, en el contexto chileno, indica que los profesores creen que la propuesta conceptual está en la base del aprendizaje significativo y en la necesidad de asegurar ciertas condiciones mínimas para que ello sea posible. La adhesión a esta dimensión reveló docentes comprometidos con los planteamientos de las actuales políticas educativas que promueven de manera paulatina y global mejorar las formas de enseñar y aprender. En este sentido, se hace hincapié en el aprendizaje más que en la enseñanza, en el conocimiento contextualizado, en la adquisición de competencias en lugar de 
acumulación de datos, en el lema preparar para la vida antes que para la universidad y capacitar para una vida de trabajo en lugar de capacitar para un empleo (Vera Bachmann \& Meneses Clavero, 2012).

Por todo esto el presente estudio, plantea como objetivos: a) analizar las propiedades psicométricas de la Escala de Creencias Acerca del Proceso Educativo en el Medio Rural (CAPE-R) en docentes de Mendoza, Argentina, b) conocer el perfil docente rural, c) comparar y analizar si existen diferencias significativas entre las creencias docentes según las variables sociodemográficas: nivel de enseñanza, antigüedad docente, cargo que ocupan, carga horaria, edad y género.

\section{Método}

\section{Participantes}

Se trabajó con una muestra no probabilística de 150 docentes (137 mujeres y 13 hombres) que dictan clases en escuelas de zonas rurales de la provincia de Mendoza (Argentina) en los niveles educativos: inicial (11.3\%), primario (34.7\%) y secundario (54\%). La edad promedio de los docentes fue $38.88 \mathrm{y}$ los años de antigüedad docente van entre $0 \mathrm{y}$ 30 años, con un promedio de 8.27. En cuanto al cargo que ocupan en la docencia, el $55.3 \%$ posee un cargo titular y el $44.7 \%$ restante poseen un cargo suplente.

\section{Medición}

Para la medición y evaluación de las variables se utilizó la Escala de Creencias Acerca del Proceso Educativo en el Medio Rural CAPE-R. La misma es una escala tipo Likert y consta de 55 ítems agrupados en cuatro dimensiones. Los ítems son creencias acerca del proceso educativo en el medio rural, por ejemplo: "Los materiales educativos entregados a la escuela favorecen el aprendizaje de los alumnos y facilitan nuestro trabajo como docentes" "Para los alumnos de zonas rurales no es importante tener un buen rendimiento académico". Se 
trata de enunciados construidos ad hoc por las autoras de la escala, en base a estudios cualitativos realizados previamente, y a investigaciones tanto teóricas como de otros instrumentos relacionados con las creencias docentes acerca del proceso educativo (Vera Bachmann et al., 2012). La escala consta de cuatro dimensiones 1) organización del proceso de enseńanza, 2) aprendizaje significativo, 3) la importancia de la práctica y experiencia de los profesores y, 4) factores que afectan el proceso de enseñanza aprendizaje. La primera dimensión refleja las creencias de que la mejor modalidad de enseñanza es la que se realiza de acuerdo al paradigma tradicional -menor flexibilidad, relaciones verticales, control autoritario, homogeneidad entre los alumnos-. La segunda dimensión, expresa las siguientes creencias: los docentes deben tener compromiso personal con la tarea, docentes y alumnos deben compartir intereses y expectativas de logro, y las actividades deben ser creativas, originales, estimulantes y buscar que tanto los alumnos como sus familias se involucren en el aprendizaje. La tercera dimensión expresa las creencias que, para alcanzar un aprendizaje significativo, es más importante la vocación docente, la experiencia y la práctica diaria que la teoría, el perfeccionamiento y la capacitación. La última dimensión muestra las creencias sobre como la enseñanza se ve obstaculizada por factores del medio contextual, tales como la cooperación de los padres, las condiciones laborales del docente, y también por factores propios del alumno como el nivel socioeconómico, el rendimiento intelectual y la disciplina.

El puntaje total para cada dimensión corresponde al puntaje promedio de los ítems que la componen y oscila entre 1 y 4 puntos. A mayor puntaje, mayor adherencia a las creencias representadas por la dimensión correspondiente

En cuanto a sus propiedades psicométricas, presenta una adecuada validez de constructo constatada a través de análisis factorial exploratorio, el cual arrojó una estructura de cuatro factores: $1 \alpha=.81$ (17 ítems); $2 \alpha=.76$ (14 ítems); $3 \alpha=.75$ (10 ítems); $4 \alpha=.73$ (14 ítems). En relación a la confiabilidad de la escala completa, el coeficiente alfa 
de Cronbach alcanzó un índice aceptable $\alpha=.88$ (Hernández Sampieri et al., 2010).

Además, se implementó una encuesta sociodemográfica diseñada ad hoc con el fin de explorar nivel de enseñanza, antigüedad docente, cargo que ocupan, carga horaria, edad, género.

\section{Procedimiento}

En un primer momento, se administró la CAPE-R a cinco docentes de una escuela rural secundaria del departamento de Rivadavia (Mendoza) con el fin de verificar que las afirmaciones resultaran comprensibles. A los fines de esta investigación se incluyó a docentes de nivel inicial y secundario ya que, estaba diseñada para docentes de escuelas primarias. Además, con el fin de ampliar el estudio, se indagaron variables como la antigüedad docente en zona rural, nivel educativo en el que se desempeña (inicial, primario o secundario), cantidad de horas cátedra dedicadas a la docencia en zona rural y cargo que actualmente ocupa (titular o suplente). En cuanto a los ítems, como adaptación lingüística, se optó por dejar las palabras completas respecto al grado de acuerdo en el cuestionario ya que en la versión original contaba con las abreviaturas MD (muy en desacuerdo), ED (en desacuerdo), EA (en acuerdo), MA (muy en acuerdo) y en nuestra población se dificultaba su entendimiento.

Luego de la prueba piloto cuyos resultados fueron satisfactorios, se tomó contacto con los directivos de 11 escuelas rurales pertenecientes a los departamentos Junín, San Martín, Las Heras, Lavalle, Luján y Maipú de la provincia de Mendoza, Argentina, para explicar el propósito y modalidad de la investigación. Una vez alcanzada la autorización correspondiente, se explicó a los docentes tanto el propósito del estudio como la modalidad de administración de la escala y se remarcó el anonimato de los datos, para luego solicitar el consentimiento informado a aquellos que deseaban participar. La toma de la muestra total se realizó de manera grupal ya que el cuestionario es autoadministrable. 


\section{Análisis de datos}

Para adaptar la escala CAPE-R se realizó un análisis factorial exploratorio utilizando el software Factor 9.2, ya que permite utilizar métodos de extracción robustos, adecuados a variables de medición ordinal, y tiende a proveer estimaciones menos sesgadas de los verdaderos valores de los parámetros (Ferrando \& Lorenzo-Seva, 2013).

Para los análisis descriptivos se utilizó el software SPSS 20.0 mediante el cual se calcularon medidas de tendencia central, coeficiente de correlación Pearson y pruebas de diferencias de medias, entre ellas prueba $t$ para muestras independientes y ANOVA.

\section{Resultados}

En primer lugar, se analizaron las propiedades psicométricas de la escala CAPE-R para medir creencias educativas de docentes rurales en nuestra población. Si bien la muestra no presentó valores perdidos ni casos atípicos univariados, la prueba de distancia de Mahalanobis $(D)$, evidenció tres casos atípicos multivariados al nivel de significación especificado $(p<.001)$. No obstante, ninguno de estos casos alcanzó un valor de influencia superior a 1.00 , por lo cual no se descartaron. Se analizó la asimetría y curtosis de cada ítem y se realizó una inspección gráfica de la distribución de los puntajes (histogramas con curva normal y gráficos Q-Q) para comprobar los supuestos de normalidad de la muestra. Un solo ítem presentó un índice de curtosis levemente superior (1.87) al punto de corte, pero se conservó dada su relevancia teórica (George \& Mallery, 2001). Para el diagnóstico de multicolinealidad entre los ítems, se analizaron los índices de tolerancia, las matrices de intercorrelación entre los ítems, y los índices de condición para cada uno de los reactivos (según los criterios propuestos por Belsely, Kuh \& Welsch, 1980, en Tabachnick \& Fidell, 2001). Los resultados en todos los casos fueron satisfactorios.

A los fines de determinar el número preliminar de factores a extraer, se realizó un primer análisis factorial exploratorio utilizando 
como método de extracción el estudio de componentes principales y se llevó a cabo el análisis paralelo de Horn (HPA, Horn, 1965). En base a los resultados obtenidos en estos estudios se realizaron nuevos análisis factoriales utilizando el como método de estimación ULS (mínimos cuadrados no ponderados), adecuado para variables ordinales.

Los 55 ítems de la escala fueron analizados mediante el análisis de Componentes Principales con el objetivo de obtener información acerca del máximo número de factores posibles de extracción y su naturaleza. El índice de adecuación muestral KMO (Kaiser-Meyer-Olkin) fue de .77, y el test de esfericidad de Bartlett fue significativo $(p<.001)$, lo cual indica una adecuada intercorrelación entre los datos y la factibilidad de realizar el análisis factorial. Se utilizó la regla de Kaiser-Gutman cuyo resultado fue una solución de 15 factores iniciales con autovalores superiores a 1, que explicaban en conjunto un $69.89 \%$ de la varianza total de la prueba. Otro criterio de selección de factores empleado fue el resultado del análisis paralelo de Horn (HPA, Horn, 1965) mediante el software Visual Stats, que permitió identificar un máximo de cuatro factores. En base a esto, se volvieron a analizar los datos utilizando el método de extracción ULS. Los resultados permitieron inferir la existencia de entre tres y cuatro factores, observándose que la solución más clara y estable fue la de cuatro.

A partir de la inspección de la matriz factorial se eliminaron los ítems con cargas factoriales menores a .40 (excepto un caso en que los valores fueron cercanos y se consideraron como indicadores relevantes del constructo), ítems con cargas compartidas (superiores a .30) con otro factor, e ítems que no correlacionaban con ningún factor. De esta manera, tal como muestra la tabla 1, se conservaron 33 ítems distribuidos en cuatro factores que explicaron un $44.19 \%$ de la varianza de respuestas al test (ver anexo). Con respecto a la consistencia interna, se encontró que el Factor 1, presentó un coeficiente alfa de .85, el Factor 2 un alfa de .76, el Factor 3, obtuvo un coeficiente .80, y el Factor 4 presentó un alfa de .74 , considerados valores buenos por la literatura científica. 


\section{Tabla 1}

Matriz de factores extraidos por Minimos cuadrados ponderados y cargas factoriales de los items

\begin{tabular}{|c|c|c|c|c|}
\hline Ítems & $\mathrm{F} 1$ & $\mathrm{~F} 2$ & F3 & F4 \\
\hline Item 10 & .56 & & & \\
\hline Item17 & .57 & & & \\
\hline Item 18 & .58 & & & \\
\hline Item 20 & .65 & & & \\
\hline Item 21 & .52 & & & \\
\hline Item31 & .51 & & & \\
\hline Item38 & .55 & & & \\
\hline Item 40 & .54 & & & \\
\hline Item41 & .68 & & & \\
\hline Item49 & .57 & & & \\
\hline Item55 & .64 & & & \\
\hline Item2 & & .29 & & \\
\hline Item 11 & & -.53 & & \\
\hline Item 15 & & -.45 & & \\
\hline Item37 & & .39 & & \\
\hline Item 43 & & -.55 & & \\
\hline Item 44 & & -.78 & & \\
\hline Item 45 & & -.52 & & \\
\hline Item47 & & .34 & & \\
\hline Item 48 & & .34 & & \\
\hline Item 50 & & -.41 & & \\
\hline Item 51 & & -.66 & & \\
\hline Item 5 & & & .58 & \\
\hline Item6 & & & .62 & \\
\hline Item8 & & & .67 & \\
\hline
\end{tabular}




\begin{tabular}{|c|c|c|c|c|}
\hline Ítems & $\mathrm{F} 1$ & $\mathrm{~F} 2$ & F3 & F4 \\
\hline Item9 & & & .47 & \\
\hline Item 12 & & & .53 & \\
\hline Item 24 & & & .58 & \\
\hline Item 25 & & & -.50 & \\
\hline Item 26 & & & .68 & \\
\hline Item 1 & & & & .69 \\
\hline Item3 & & & & .65 \\
\hline Item4 & & & & .75 \\
\hline$\alpha$ & .85 & .76 & .80 & .74 \\
\hline
\end{tabular}

En segundo término, con el objetivo de conocer cuáles son las creencias docentes de los profesores rurales en nuestra población, se redefinieron las cuatro dimensiones como se observa en la tabla 2 y se obtuvo el promedio y desvío estándar de cada una. Se encontró que la dimensión 4 "dificultades en el proceso educativo" alcanzó una media de 2.83. Entre tanto, la dimensión 1 "factores que influyen en el proceso educativo" alcanzó una media de 2.69 y la segunda dimensión "Mediadores externos para el aprendizaje o agentes de apoyo externo" obtuvo una media de 2.46. La media de la dimensión 3 "modalidad y experiencia docente" alcanzó el menor promedio 2.07 .

\section{Tabla 2}

Creencias docentes acerca del proceso educativo

\begin{tabular}{lcc}
\hline Dimensiones & $M$ & $D E$ \\
\hline Factores que influyen en el proceso educativo & 2.69 & .46 \\
Mediadores externos para el aprendizaje & 2.46 & .28 \\
Modalidad y experiencia docente & 2.07 & .37 \\
Dificultades en el proceso educativo & 2.83 & .69 \\
\hline
\end{tabular}


Por último, se realizó un análisis de las diferencias entre las creencias docentes según las variables sociodemográficas: nivel educativo en el que dicta las clases, antigüedad docente, cargo docente, dedicación docente, edad y género. El análisis correlacional arrojó que no existe correlación entre las dimensiones de la escala CAPE-R y la edad de los profesores: (1. $r=-.075 ; p=.359)(2 . r=-.003 ; p=.974)(3 . r=.045$; $p=.586)$ (4. $r=-.003 ; p=.974)$, así como tampoco con la antigüedad en docencia: (1. $r=-.075 ; p=374)(2 . r=.041 ; p=.626)(3 . r=.104$; $p=.216)(4 . r=.049 ; p=.561)$. No se encontró relación entre la dedicación docente entendida en cantidad de horas catedra y, los componentes de la escala (1. $r=-.020 ; p=.828)(2 . r=-.042 ; p=.650)(3 . r=.125$; $p=.181)(4 . r=-.019 ; p=.837)$.

El análisis de diferencia de medias ( $t$ de student) no indicó diferencias significativas entre el género y el cargo docente titular o suplente con las dimensiones del CAPE-R tal como se muestra en la tabla 3.

\section{Tabla 3}

Comparación de medias de las creencias docentes según el género y cargo que ocupan

\begin{tabular}{cccccccccc}
\hline & Fem. & Masc. & & \multicolumn{5}{c}{ Tit. } & Supl. \\
& $n=137$ & $n=13$ & & & $n=83$ & $n=67$ & & \\
& $M$ & $M$ & $t$ & $p$ & $M$ & $M$ & $t$ & $p$ \\
\hline Dimensión 1 & 29.70 & 28.76 & -.63 & .528 & 29.74 & 29.47 & .32 & .749 \\
Dimensión 2 & 27.01 & 27.69 & .75 & .454 & 27.24 & 26.86 & .73 & .464 \\
Dimensión 3 & 16.63 & 16.15 & -.55 & .581 & 16.91 & 16.19 & 1.47 & .143 \\
Dimensión 4 & 8.58 & 7.61 & -1.61 & .109 & 8.48 & 8.52 & -.11 & .906 \\
\hline
\end{tabular}

Para estudiar la asociación entre la variable nivel educativo en el que dicta clases y las dimensiones del cuestionario se implementó ANOVA de un factor. A partir de la lectura de datos se observa que no existen diferencias significativas en las dimensiones $1(p=.75), 2$ 
$(p=.10)$ y $3(p=.08)$ en relación al nivel en el que el docente dicta clases; contrario a los resultados hallados en la dimensión $4(p=.01)$. Para precisar en qué medida difieren los grupos estudiados se utilizó como análisis post-hoc la prueba de Bonferroni donde se constató que las diferencias en cuanto a las creencias se encuentran dentro de los niveles inicial y primario $(p<.01)$.

\section{Tabla 4}

ANOVA de las dimensiones de creencias docentes según el nivel educativo donde dictan clases

\begin{tabular}{|c|c|c|c|c|c|c|c|c|}
\hline & \multicolumn{2}{|c|}{$\begin{array}{c}\text { Inicial } \\
(n=17)\end{array}$} & \multicolumn{2}{|c|}{$\begin{array}{c}\text { Primario } \\
(n=52)\end{array}$} & \multicolumn{2}{|c|}{$\begin{array}{l}\text { Secundario } \\
\quad(n=81)\end{array}$} & \multirow[b]{2}{*}{$F$} & \multirow[b]{2}{*}{$p$} \\
\hline & $M$ & $D E$ & $M$ & $D E$ & $M$ & $D E$ & & \\
\hline Dimensión 1 & 29.29 & 5.25 & 29.28 & 3.98 & 29.91 & 5.71 & .276 & .75 \\
\hline Dimensión 2 & 26.17 & 2.48 & 26.61 & 2.89 & 27.55 & 3.29 & 2.29 & .10 \\
\hline Dimensión 3 & 15.29 & 2.56 & 16.40 & 2.85 & 16.98 & 3.10 & 2.45 & .08 \\
\hline Dimensión 4 & 7.29 & 1.86 & 9.01 & 1.89 & 8.41 & 2.13 & 4.77 & .01 \\
\hline
\end{tabular}

\section{Discusión}

El propósito final de este artículo fue implementar la escala CAPE-R en docentes rurales de Mendoza, Argentina. Para ello, en primer lugar se verificó que los ítems cumplan con los criterios de normalidad. Si bien, el análisis factorial mostró 4 factores al igual que la escala original, la retención de los ítems produjo una excesiva supresión de los mismos: de 55 ítems que componían la versión original se obtuvieron solo 33 ítems. Además, muchos ítems se agruparon de manera disímil a la escala original. De este modo, se redefinió la interpretación de cada factor como se detalla a continuación. La dimensión 1 se denominó "factores que influyen en el proceso educativo", la cual agrupa las 
creencias sobre el proceso de enseñar y aprender como intrínsecamente vinculado tanto a factores disciplinares y motivacionales propios de los alumnos como la experiencia del docente y factores socioeconómicos traducidos en agentes positivos o de riesgo. Algunas de los ítems que componen esta dimensión son "La calidad del conocimiento de los alumnos depende de la cantidad de la experiencia de los docentes" "Los docentes que trabajan en zonas rurales con mayor experiencia conocen las características de los alumnos, por lo tanto pueden enseñarles de un modo más adecuado" "El bajo nivel socioeconómico de los alumnos influye negativamente en el aprendizaje”. En la segunda dimensión "mediadores externos para el aprendizaje", convergen todos los ítems relacionados a las creencias sobre la importancia de materiales educativos, capacitaciones, y cooperación entre comunidad, docentes, padres y alumnos para un buen desempeño educativo.

Además, este factor, refleja que los componentes socio-ambientales y conductuales afectan el nivel de aprendizaje. "Para los alumnos de zonas rurales no es importante tener un buen rendimiento académico", "Actualmente, no existe cooperación entre maestros/as y alumnos en zonas rurales", son algunos descriptores de esta dimensión. La tercera dimensión "modalidad y experiencia docente" expresa la importancia de la disciplina y castigos como una práctica de enseñanza "Para controlar al grupo en el curso son más eficaces los castigos, notas en los cuadernos y llamadas de atención, que los refuerzos y premios” y, refleja la desestima y desilusión por la actividad docente rural así como el descreimiento en la capacidad de los alumnos "Un docente que dicta clases flexibles dificulta el buen rendimiento de los alumnos". La cuarta dimensión "dificultades en el proceso educativo" abarca las creencias de que las dificultades enfrentadas en el proceso de enseńanza-aprendizaje son causadas principalmente debido a la falta de compromiso por parte de los alumnos y al desinterés de las familias acerca de la enseñanza. Esta dimensión se conforma solo de tres ítems "Ninguna estrategia de aprendizaje lleva a un buen rendimiento, si no se logra el compromiso de parte del alumno", "Los docentes sabemos y aceptamos que no todos los alumnos alcanzarán las metas educativas" y "La mayoría de 
las dificultades enfrentadas en el proceso de aprendizaje de los alumnos, se deben al desinterés que existe en las familias acerca de los procesos de enseñanza”.

Como se observa en los resultados, la escala posee adecuadas propiedades de validez y confiabilidad, obtenidas a partir de los criterios que establecen los estándares para la evaluación educativa y psicológica (AERA/APA/NCME, 2014), por lo cual se constituye como una herramienta valiosa a tener en cuenta para futuras investigaciones sobre la temática.

En cuanto al perfil docente en nuestra población argentina, se encontraron valoraciones medias mayores para la dimensión 4 "dificultades en el proceso educativo", lo cual revela que los docentes rurales argentinos creen que el desarrollo del trayecto educativo se encuentra afectado por la desmotivación familiar e individual del alumno más que por cuestiones exclusivamente escolares. Estos resultados coinciden con los encontrados por Castellanos Simons et al (2015) quienes sostienen que los alumnos de alta capacidad intelectual del contexto rural quedan en franca desventaja de los alumnos de escuelas urbanas en cuanto al apoyo familiar. Otra explicación posible, son las desigualdades socioeconómicas y educativas. La reciente investigación llevada a cabo por Guadagni y Boero (2015) sostiene que existe una alta proporción (más del 50\%) de los adolescentes que no completan el ciclo secundario, y en su mayoría se corresponden con los sectores menos favorecidos y rurales.

En contraposición con el estudio original de la Escala, esta valoración posiblemente refleje el desamparo que viven los docentes argentinos traducido en el concepto de malestar docente que habita la contemporaneidad (Schiavetta, 2013). Los cambios socioeducativos promueven constantemente nuevas exigencias y funciones, generan en el docente desconcierto ante las demandas y exigencias extra e intrainstitucionales cada vez más antagónicas que lo sumergen en diferentes escenarios para los cuales no fue formado. Pensar al docente como garante de la formación y la integridad de los alumnos, a costa de su propia integridad, produce la negación de su situación de sufrimiento y de desamparo (Duschatzky, 2007). 
En cuanto a las variables sociodemográficas y las dimensiones que arroja la escala, no existen diferencias significativas. Contrariamente a los resultados de Vera Bachmann y Meneses Clavero (2012) este estudio coincidió con un trabajo llevado a cabo en España donde no se encontraron relaciones entre la edad y los perfiles de creencias estudiados (Molpecereset al 2004). Además, el estudio original, evidenció que las creencias que sustentan los profesores con menor edad están relacionadas con parámetros más acordes a las innovaciones pedagógicas actuales, las que al mismo tiempo son más nuevas para los profesores de mayor edad, quienes poseen arraigadas creencias referidas a medidas más convencionales y menos flexibles para realizar su práctica pedagógica.

En relación a la variable antigüedad en la docencia en zona rural, se puede afirmar que no hay diferencias estadísticamente significativas. Estos resultados discrepan de otros estudios que afirman que los docentes con mayor experiencia muestran una mayor preocupación en relación con los contenidos didácticos, mientras que los docentes con menor experiencia se focalizan mayoritariamente en la gestión del aula y tendrían menos habilidad en el conocimiento del contenido desde la perspectiva del estudiante, el conocimiento profundo del contenido, conocer cómo presentar el contenido de manera apropiada y conocer cómo integrar el aprendizaje con objetivos curriculares más amplios (Díaz et al., 2010). En el estudio realizado por Vera Bachmann y Meneses Clavero (2012) encontraron una relación similar entre los años de antigüedad en la docencia en zona rural y la edad del docente, respecto a que los docentes con menor cantidad de ańos de ejercicio profesional también tienden a rechazar las creencias relacionadas con tres dimensiones. Cabe señalar que respecto a la correlación entre la cantidad de horas trabajadas por los docentes y las creencias, no se han encontrado antecedentes que evalúen dicha asociación, sin embargo desde este estudio se aporta que no se ha evidenciado alguna correlación significativa, es decir que las creencias que adopten los docentes son independientes de la cantidad de horas que trabaje.

En el caso de la asociación entre el género y las creencias docentes, aunque los hombres puntúan de forma superior en las dimensiones 
1 y 2 y las mujeres puntúan por encima en las dimensiones 3 y 4; se observó que la diferencia de medias no es significativa (Vera Bachmann \& Meneses Clavero, 2012; Molpeceres et al., 2004). Sin embargo, los estadísticos descriptivos muestran que los hombres puntúan de forma superior en las dimensiones 1 "factores que influyen en el proceso educativo" y 2 "mediadores externos que impactan en el aprendizaje", y las mujeres obtuvieron valores mayores en las dimensiones 3 "modalidad y experiencia docente" y 4 "dificultades en el proceso educativo". Esta discrepancia posiblemente responda a la desigualdad muestral en ambos géneros, ya que se trabajó con 137 mujeres y solo 13 hombres. Esta disparidad no puede dejar de asociarse a la presencia prioritariamente femenina en el nivel docente. Según los datos publicados en el Censo Nacional del Personal de los Establecimientos Educativos 2014 sobre un total de 732819 docentes, solo 151304 son varones (Ministerio de Educación de la Nación, 2014).

En el caso del cargo que ocupa el docente, es decir, si trabaja en condición de titular o suplente, tampoco se ha evidenciado alguna relación significativa entre estas condiciones contractuales y las creencias docentes, coincidiendo con estudios previos (Molpeceres et al., 2004). Por último, se encontró una diferencia significativa entre el nivel de enseñanza docente, es decir nivel inicial, primario y secundario, con las creencias docentes. Las relaciones se aprecian en la dimensión 4 "dificultades en el proceso educativo" la cual tiene que ver, como anteriormente se mencionó, con la creencia de que las dificultades enfrentadas en el proceso educativo se deben principalmente, a la falta de compromiso por parte de los alumnos y al desinterés de las familias acerca de la enseńanza. Luego de realizar la prueba post-hoc, se observó que las diferencias en cuanto a los niveles de enseñanza se encontraban principalmente dentro de los niveles inicial y primario. De acuerdo con este punto, Jiménez Llanos (2005), agrega que en los niveles inicial y primario hay una tendencia a relaciones entre profesor y alumno que se enmarcaría dentro de las teorías conservadoras.

En coincidencia con Vera Bachmann y su equipo (2012) estos resultados podrían contribuir a dar respuesta a uno de los principales 
objetivos del modelo educativo rural: favorecer la diversidad de sus actores, tanto en lo cultural, como en lo social y étnico. De este modo, la falta de concordancia entre la estructura factorial original y la hallada en este análisis amerita la realización de un análisis factorial confirmatorio con muestras de mayor tamaño. Si bien, es importante remarcar que la muestra debería haber sido mayor. No obstante, estudios recientes (Lloret Segura, Ferreres Traver, Hernández Baeza \& Tomas Marco, 2014) afirman que estos criterios clásicos son desaconsejables, y que, no existe una receta clara, ya que el tamaño mínimo recomendado depende de una serie de factores como lo son el número de ítems que definen el factor, la homogeneidad de la muestra y, muy especialmente, la comunalidad de los ítems. Lógicamente, cuanto mayor sea el tamaño muestral, más confianza se tendrá (Ferrando \& Anguiano Carrasco, 2010; Lloret Segura et al., 2014). Asimismo, el análisis psicométrico de la escala CAPE-R representa un avance tanto para la evaluación psicológica como para la validación de instrumentos confiables para, luego, utilizarlos en estudios de mayor amplitud.

Se considera fundamental poder complementar este estudio con otros instrumentos, que permitan profundizar las investigaciones sobre la compleja relación entre docentes-alumnos y los procesos de enseñanza-aprendizaje. De este modo, ampliar el estudio sobre cómo las creencias docentes se arraigan en el quehacer diario o si se han podido cambiar. Se recomienda también realizar estudios similares que abarquen o amplíen observaciones, datos que en este estudio no se han podido contemplar, como por ejemplo, la comparación entre los distintos departamentos de Mendoza, el cotejo de diferencias entre zonas disímiles como lo son las zonas urbanas, marginales, a cuál de las dimensiones adhieren más los docentes, etc.

Para concluir, se puede pensar que se ha logrado vislumbrar la relación entre los docentes de nuestras escuelas rurales de Mendoza y sus creencias, de este modo se considera que los resultados alcanzados conforman una base empírica para la promoción de técnicas adaptadas a nuestro medio y, de este modo, contribuir al avance dentro del ámbito de la evaluación en psicología. 
Propiedades psicométricas de la Escala CAPE-R en docentes de Mendoza, Argentina / Labin et al.

\section{Referencias}

American Educational Research Association, American Psychological Association, \& National Council of Measurement in EducationAERA, APA, NCME. (2014). The standards for educational and psychological testing. Washington, DC: AERA

Blanco, G. (Ed.) (2007). Ejemplos para pensar la enseñanza en plurigrado. Recuperado de http://www.me.gov.ar/curriform/publica/ plurigrado.pdf

Castellanos Simons, D., Ferrari Belmont, A. M., Hernández Rodríguez, C. A. \& Bazán Ramírez, A. (2015). Apoyo familiar en escolares de alta capacidad intelectual de diferentes contextos socioeducativos. Revista de Psicología, 33(2), 299-332. Recuperado de http://revistas.pucp.edu.pe/index.php/psicologia/ article/view/13046/13659

Contreras Palma, S. (2008). Qué piensan los profesores sobre sus clases: estudio sobre las creencias curriculares y las creencias de actuación curricular. Formación Universitaria, 1(3), 3-11. https://doi. org/10.4067/S0718-50062008000300002

Díaz, M., Martínez P., Roa I. \& Sanhueza M. (2010). Los docentes en la sociedad actual: sus creencias y cogniciones pedagógicas respecto al proceso didáctico. Revista de la Universidad Bolivariana, 25(9), 421-436. https://doi.org/10.4067/ S0718-65682010000100025

Dirección General de Cultura y Educación (2007). La tarea de enseñar en el ámbito rural. Recuperado de http://servicios2.abc.gov.ar/ lainstitucion/sistemaeducativo/educacioninicial/capacitacion/ documentoscirculares/2007/ambitosrurales.pdf

Duschatzky, S. (2007). Maestros errantes. Experiencias sociales a la intemperie. Buenos Aires: Paidós.

Fairstein, G. \& Pedernera, A. (2007). El plurigrado: una práctica pedagógica singular. Argentina. Educare, 2, 33-42. Recuperado de http://revistas.una.ac.cr/index.php/EDUCARE/article/ view/1374 
Fernández Nistal, M., Perez Ibarra, R., Tuset Bertrán, A. \& García Hernández, C. (2013). Prácticas educativas y creencias de profesores de secundaria pertenecientes a escuelas de diferentes contextos socioeconómicos. Perfiles Educativos, 35(139), 40-59. https:// doi.org/10.1016/S0185-2698(13)71808-7

Ferrando, P. \& Anguiano-Carrasco, C. (2010). El análisis factorial como técnica de investigación en psicología. Papeles del Psicólogo, 31(1), 18-33. Recuperado de http://www.papelesdelpsicologo. es/pdf/1793.pdf

Ferrando, P. J., \& Lorenzo Seva, U. (2013). Unrestricted item factor analysis and some relations with item response theory. Technical report. Department of Psychology, Universitat Rovirai Virgili, Tarragona. Recuperado de http://psico.fcep.urv.es/utilitats/factor.

George, D. \& Mallery, M. P. (2001). SPSS for Windows step by step: A simple guide and reference. Boston, MA: Allyn \& Bacon.

Guadagni, A. A. \& Boero, F. (2015). La educación argentina en el siglo XXI. Buenos Aires: El Ateneo Grupo Impresor S.A.

Hernández Sampieri, R., Fernández Collado y Lucio C. B. (2010). Metodología de la investigación. (5 Ed.). México: Mc Graw-Hill Interamericana Editores.

Horn, J. L. (1965). A rationale and test for the number of factors in a factor analysis. Psychometrika, 30, 179-185. https://doi. org/10.1007/BF02289447

Jiménez Llanos, A. (2005). Las teorías implícitas del profesorado sobre los distintos ámbitos del proceso de enseńanza-aprendizaje: la interacción profesor-alumno en los tres niveles educativos. Qurriculum: Revista de teoría, investigación y práctica educativa, (18), 211-236. Recuperado de http://publica.webs.ull.es/ upload/REV\%20QURRICULUM/18\%20-\%202005/10\%20 (Ana\%20Beatriz\%20Jim\%C3\%A9nez\%20Llanos).pdf

Lloret Segura, S., Ferreres Traver, A, Hernández Baeza, A. \& Tomas Marco, I. (2014). El análisis factorial exploratorio de los ítems: una guía práctica, revisada y actualizada. Anales de psicología, 30(3), 1151-1169. https://doi.org/10.6018/analesps.30.3.199361 
López Vargas B. \& Basto Torrado S. (2010). Desde las teorías implícitas a la docencia como práctica reflexiva. Educación y Educadores, 13(2), 275-291. https://doi.org/10.5294/edu.2010.13.2.6

Mansilla Sepúlveda, J. \& Beltrán Véliz, J. (2013). Coherencia entre las estrategias didácticas y las creencias curriculares de los docentes de segundo ciclo, a partir de las actividades didácticas. Perfiles Educativos, 35(139), 25-39. https://doi.org/10.1016/ S0185-2698(13)71807-5

Ministerio de Educación de la Nación. (2014) Censo Nacional del Personal de los Establecimientos Educativos, Argentina. Recuperado de http://portales.educacion.gov.ar/diniece/censo-docente/

Molpeceres, M., Chulvi, B. \& Bernad, J. (2004). Concepciones sobre la enseñanza y prácticas docentes en un sistema educativo en transformación. Universidad de Valencia. Recuperado de http://www. ilo.org/public//spanish/region/ampro/cinterfor/publ/molpece/ pdf/mochube.pdf

Myers, D. (2000). Psicología Social. Bogotá, Colombia: Mc Graw Hill Netzahualcoyotl-Netzahual, M. (2015). Las representaciones sociales de los docentes sobre la Reforma Integral de la Educación Básica. Revista iberoamericana de educación superior, 6(15), 3-25. https://doi.org/10.1016/S2007-2872(15)30001-9

Pozo Municio, J.I., Pérez Echeverría, M., Martín Ortega, E., Scheuer Rubiños, N., De la Cruz, M. \& Mateos Sanz, M. (2006). Nuevas formas de pensar la enseñanza y el aprendizaje: Las concepciones de profesores y alumnos. Barcelona: Graó.

Prieto, M. (2008). Creencias de los profesores sobre Evaluación y Efectos Incidentales. Revista de Pedagogía, 29(84), 123-144. Recuperado de http://beceneslp.edu.mx/PLANES2012/4o\%20 Sem/02\%20Evaluaci\%F3n\%20para\%20el\%20aprendizaje/ Materiales/PDF/art05b.pdf

Rodrigo, M. J., Rodríguez, A. \& Marrero, J. (1993). Las teorías implicitas: una aproximación al conocimiento cotidiano. Madrid: Visor. Rosbaco, I. C. (2007). El docente como representante del otro social: su función subjetivante. Revista de la Escuela de Ciencias de 
la Educación, 2, 161-177. Recuperado de http://www.revistacseducacion.unr.edu.ar/ojs/index.php/educacion/article/ view/139/137

Schiavetta, L. (2013). Sobre el docente y el malestar en la modernidad tardía. En A. Taborda y G. Leoz (Comps.), Psicología Educacional en el Contexto de la Clínica Socioeducativa Vol. II. (pp. 167-189). Recuperado de: http://www0.unsl.edu.ar/ disgraf/ neuweb2/pdf/Taborda\%20Volumen1.pdf

Tabachnick, B. \& Fidell, L. (2001). Using multivariate statistics (4th ed.). New York: Harper \& Row.

Vera Bachmann, D. \& Meneses Clavero, P. (2012). Construcción de una escala para medir creencias acerca del proceso educativo en profesores rurales, CAPE-R. Liberabit. Revista de psicología, 18(2), 183-193. Recuperado de http://www.redalyc.org/articulo.oa? id=68624684009

Vera Bachmann, D., Osses, S. \& Fuenzalida, E. (2012). Las creencias de los profesores rurales: una tarea pendiente para la investigación educativa. Estudios Pedagógicos, 1, 297-310. https://doi. org/10.4067/S0718-07052012000100018

Recibido: 13 de mayo, 2016

Revisado: 13 de agosto, 2017 Aceptado: 03 de octubre, 2017 


\section{Apéndice}

\section{Escala de Creencias Acerca del Proceso Educativo en el Medio Rural (CAPE-R) \\ Mendoza, Argentina}

Sexo: ( ) Masculino ( ) Femenino

Edad:

Antigüedad en la docencia en zona rural: años

Nivel de enseñanza: Inicial ( ) Primario ( ) Secundario ( )

Cantidad de horas cátedra dedicadas a la docencia en zona rural:

Cargo: Titular ( ) Suplente ( )

RECUERDE QUE ES MUY IMPORTANTE SU OPINIÓN Y QUE CONTESTE A TODAS LAS PREGUNTAS. MUCHAS GRACIAS.

\begin{tabular}{|l|l|l|l|l|l|}
\hline & $\begin{array}{c}\text { Muy en } \\
\text { desacuerdo }\end{array}$ & $\begin{array}{c}\text { En } \\
\text { desacuerdo }\end{array}$ & $\begin{array}{c}\text { En } \\
\text { acuerdo }\end{array}$ & $\begin{array}{c}\text { Muy en } \\
\text { acuerdo }\end{array}$ & $\begin{array}{c}\text { No entendí } \\
\text { la consigna }\end{array}$ \\
\hline $\begin{array}{l}\text { 1. Los docentes sabemos } \\
\text { y aceptamos que no todos } \\
\text { los alumnos alcanzarán las } \\
\text { metas educativas. }\end{array}$ & & & & & \\
\hline $\begin{array}{l}\text { 2. Los materiales edu- } \\
\text { cativos entregados a la } \\
\text { escuela favorecen el apren- } \\
\text { dizaje de los alumnos y } \\
\text { facilitan nuestro trabajo } \\
\text { como docentes. }\end{array}$ & & & & & \\
\hline $\begin{array}{l}\text { 3. La mayoría de las difi- } \\
\text { cultades enfrentadas en el } \\
\text { proceso de aprendizaje de } \\
\text { los alumnos, se deben al } \\
\text { desinterés que existe en las } \\
\text { familias acerca de los pro- } \\
\text { cesos de enseñanza. }\end{array}$ & & & & & \\
\hline
\end{tabular}




\begin{tabular}{|l|l|l|l|l|l|}
\hline & $\begin{array}{c}\text { Muy en } \\
\text { desacuerdo }\end{array}$ & $\begin{array}{c}\text { En } \\
\text { desacuerdo }\end{array}$ & $\begin{array}{c}\text { En } \\
\text { acuerdo }\end{array}$ & $\begin{array}{c}\text { Muy en } \\
\text { acuerdo }\end{array}$ & $\begin{array}{c}\text { No entendí } \\
\text { la consigna }\end{array}$ \\
\hline $\begin{array}{l}\text { 4. Ninguna estrategia de } \\
\text { aprendizaje lleva a un buen } \\
\text { rendimiento, si no se logra } \\
\text { el compromiso de parte del } \\
\text { alumno. }\end{array}$ & & & & \\
\hline $\begin{array}{l}\text { 5. Para trabajar en el medio } \\
\text { rural, no es necesario que } \\
\text { los profesores nos perfec- } \\
\text { cionemos constantemente. }\end{array}$ & & & & \\
\hline $\begin{array}{l}\text { 6. A pesar de sus capaci- } \\
\text { dades, la mayoría de los } \\
\text { alumnos no logra los obje- } \\
\text { tivos académicos que los } \\
\text { profesores esperamos. }\end{array}$ & & & & \\
\hline $\begin{array}{l}\text { 7. Un docente que dicta } \\
\text { clases flexibles dificulta el } \\
\text { buen rendimiento de los } \\
\text { alumnos. }\end{array}$ & & & & \\
\hline $\begin{array}{l}\text { 8. La educación en el } \\
\text { medio rural se dificulta por } \\
\text { la existencia de ciertas cos- } \\
\text { tumbres, normas yalores } \\
\text { propios de este medio. }\end{array}$ & & & & \\
\hline $\begin{array}{l}\text { 9. La calidad del cono- } \\
\text { cimiento de los alumnos } \\
\text { depende de la cantidad } \\
\text { de la experiencia de los } \\
\text { docentes. }\end{array}$ & & & & \\
\hline $\begin{array}{l}\text { 10. Actualmente, no existe } \\
\text { cooperación entre maes- } \\
\text { tros/as y alumnos en zonas } \\
\text { rurales. }\end{array}$ & & & & \\
\hline
\end{tabular}


Propiedades psicométricas de la Escala CAPE-R en docentes de Mendoza, Argentina / Labin et al.

\begin{tabular}{|l|l|l|l|l|l|}
\hline & $\begin{array}{c}\text { Muy en } \\
\text { desacuerdo }\end{array}$ & $\begin{array}{c}\text { En } \\
\text { desacuerdo }\end{array}$ & $\begin{array}{c}\text { En } \\
\text { acuerdo }\end{array}$ & $\begin{array}{c}\text { Muy en } \\
\text { acuerdo }\end{array}$ & $\begin{array}{c}\text { No entendí } \\
\text { la consigna }\end{array}$ \\
\hline $\begin{array}{l}\text { 11. Lograr que alumnos } \\
\text { de ambientes socioeconó- } \\
\text { micos bajos aprendan, no } \\
\text { es nuestra responsabilidad } \\
\text { como docentes. }\end{array}$ & & & & & \\
\hline $\begin{array}{l}\text { 12. La evaluación debe ser } \\
\text { igual para todos, solo así se } \\
\text { es justo. }\end{array}$ & & & & & \\
\hline $\begin{array}{l}\text { 13. Los alumnos aplicados } \\
\text { alcanzan más logros que } \\
\text { los menos esforzados, con } \\
\text { independencia de cuanto } \\
\text { nos dediquemos los profe- } \\
\text { sores a motivarlos. }\end{array}$ & & & & \\
\hline $\begin{array}{l}\text { 14. Los docentes que } \\
\text { trabajan en zonas rurales } \\
\text { con mayor experiencia } \\
\text { conocen las características } \\
\text { de los alumnos, por lo } \\
\text { tanto pueden enseñarles de } \\
\text { un modo más adecuado. }\end{array}$ & & & & \\
\hline $\begin{array}{l}\text { 15. Si los alumnos no } \\
\text { tienen hábitos de disciplina } \\
\text { en su hogar, no aceptarán } \\
\text { la disciplina en la escuela. }\end{array}$ & & & & \\
\hline $\begin{array}{l}\text { 16. En la actualidad, el } \\
\text { principal objetivo de la } \\
\text { educación que se lleva a } \\
\text { cabo en la zona rural, es } \\
\text { motivar y preparar a los } \\
\text { alumnos para continuar } \\
\text { sus estudios de enseñanza } \\
\text { media. }\end{array}$ & & & & \\
\hline $\begin{array}{l}\text { 17. La nota máxima es solo } \\
\text { para unos pocos alumnos. }\end{array}$ & & & & \\
\hline
\end{tabular}




\begin{tabular}{|c|c|c|c|c|c|}
\hline & $\begin{array}{c}\text { Muy en } \\
\text { desacuerdo }\end{array}$ & $\begin{array}{c}\text { En } \\
\text { desacuerdo }\end{array}$ & $\begin{array}{c}\text { En } \\
\text { acuerdo }\end{array}$ & $\begin{array}{l}\text { Muy en } \\
\text { acuerdo }\end{array}$ & $\begin{array}{l}\text { No entendí } \\
\text { la consigna }\end{array}$ \\
\hline $\begin{array}{l}\text { 18. Las actividades de } \\
\text { aprendizaje en el aula } \\
\text { deben ser diseñadas de } \\
\text { modo que todos los } \\
\text { alumnos de la clase puedan } \\
\text { realizarlas. }\end{array}$ & & & & & \\
\hline $\begin{array}{l}\text { 19. Para controlar al grupo } \\
\text { en el curso son más eficaces } \\
\text { los castigos, notas en los } \\
\text { cuadernos y llamadas de } \\
\text { atención, que los refuerzos } \\
\text { y premios. }\end{array}$ & & & & & \\
\hline $\begin{array}{l}\text { 20. Los alumnos que tienen } \\
\text { altos niveles de motivación, } \\
\text { alcanzan rendimientos aca- } \\
\text { démicos elevados. }\end{array}$ & & & & & \\
\hline $\begin{array}{l}\text { 21. Una comunidad com- } \\
\text { prometida, es la mejor } \\
\text { herramienta que los } \\
\text { docentes tenemos para des- } \\
\text { empeñarnos efectivamente. }\end{array}$ & & & & & \\
\hline $\begin{array}{l}\text { 22. Cualquier innovación } \\
\text { educativa, para tener } \\
\text { éxito, debe surgir de la } \\
\text { experiencia de los docentes } \\
\text { en la zona rural. }\end{array}$ & & & & & \\
\hline $\begin{array}{l}\text { 23. La enseñanza de los } \\
\text { alumnos que asisten a } \\
\text { escuelas rurales y que } \\
\text { poseen necesidades educa- } \\
\text { tivas especiales solo puede } \\
\text { estar en manos de docentes } \\
\text { especialistas. }\end{array}$ & & & & & \\
\hline
\end{tabular}


Propiedades psicométricas de la Escala CAPE-R en docentes de Mendoza, Argentina / Labin et al.

\begin{tabular}{|l|l|l|l|l|l|}
\hline & $\begin{array}{c}\text { Muy en } \\
\text { desacuerdo }\end{array}$ & $\begin{array}{c}\text { En } \\
\text { desacuerdo }\end{array}$ & $\begin{array}{c}\text { En } \\
\text { acuerdo }\end{array}$ & $\begin{array}{c}\text { Muy en } \\
\text { acuerdo }\end{array}$ & $\begin{array}{c}\text { No entendí } \\
\text { la consigna }\end{array}$ \\
\hline $\begin{array}{l}\text { 24. Un alumno que tiene } \\
\text { problemas conductuales, } \\
\text { también tiene problemas } \\
\text { de rendimiento. }\end{array}$ & & & & & \\
\hline $\begin{array}{l}\text { 25. Los alumnos más } \\
\text { lentos nos impiden, a los } \\
\text { docentes, avanzar en el } \\
\text { proceso de enseñanza. }\end{array}$ & & & & & \\
\hline $\begin{array}{l}\text { 26. Para los alumnos } \\
\text { de zonas rurales no es } \\
\text { importante tener un buen } \\
\text { rendimiento académico. }\end{array}$ & & & & \\
\hline $\begin{array}{l}\text { 27. La sala de clases es } \\
\text { irremplazable como } \\
\text { escenario para desarrollar } \\
\text { el proceso de enseñanza. }\end{array}$ & & & & \\
\hline $\begin{array}{l}\text { 28. El apoyo de la } \\
\text { comunidad favorece el } \\
\text { desarrollo del proceso } \\
\text { educativo. }\end{array}$ & & & & \\
\hline $\begin{array}{l}\text { 29. Los objetivos de } \\
\text { laducación actual } \\
\text { son incompatibles con } \\
\text { los estilos antiguos de } \\
\text { enseñanza, por lo tanto es } \\
\text { necesario que los docentes } \\
\text { estemos en constante } \\
\text { capacitación. }\end{array}$ & & & & \\
\hline $\begin{array}{l}30 . \quad \text { El bajo nivel } \\
\text { socioeconómico ico } \\
\text { los alumnos influye } \\
\text { negativamente en el } \\
\text { aprendizaje. }\end{array}$ & & & & \\
\hline
\end{tabular}




\begin{tabular}{|l|l|l|l|l|l|}
\hline & $\begin{array}{c}\text { Muy en } \\
\text { desacuerdo }\end{array}$ & $\begin{array}{c}\text { En } \\
\text { desacuerdo }\end{array}$ & $\begin{array}{c}\text { En } \\
\text { acuerdo }\end{array}$ & $\begin{array}{c}\text { Muy en } \\
\text { acuerdo }\end{array}$ & $\begin{array}{c}\text { No entendí } \\
\text { la consigna }\end{array}$ \\
\hline $\begin{array}{l}\text { 31. El rendimiento de } \\
\text { los alumnos depende } \\
\text { exclusivamente de sus } \\
\text { características personales. }\end{array}$ & & & & \\
\hline $\begin{array}{l}\text { 32. Los alumnos con } \\
\text { problemas de conducta no } \\
\text { aprenden y no es nuestra } \\
\text { labor disciplinarlos para } \\
\text { que lo hagan. }\end{array}$ & & & & & \\
\hline $\begin{array}{l}\text { 33. La experiencia y años } \\
\text { de práctica del docente, } \\
\text { son fundamentales para un } \\
\text { desempeńo favorable de } \\
\text { los alumnos. }\end{array}$ & & & & & \\
\hline
\end{tabular}

